\title{
DEFINITIONS OF FINITENESS BASED ON ORDER PROPERTIES
}

\author{
OMAR DE LA CRUZ, DAMIR D. DZHAFAROV, AND ERIC J. HALL
}

July 24,2004

\begin{abstract}
A definition of finiteness is a set-theoretical property of a set that, if the Axiom of Choice ( $\mathrm{AC}$ ) is assumed, is equivalent to stating that the set is finite; several such definitions have been studied over the years. In this article we introduce a framework for generating definitions of finiteness in a systematical way: basic definitions are obtained from properties of certain classes of binary relations, and further definitions are obtained from the basic ones by closing them under subsets or under quotients.

We work in set theory without AC to establish relations of implication and independence between these definitions, as well as between them and other notions of finiteness previously studied in the literature. It turns out that several well known definitions of finiteness (including Dedekind finiteness) fit into our framework by being equivalent to one of our definitions; however, a few of our definitions are actually new. We also show that Ia-finite unions of Ia-finite sets are P-finite (one of our new definitions), but that the class of P-finite sets is not provably closed under union.
\end{abstract}

\section{INTRODUCTION}

It is well known that several characterizations of finiteness fail to be equivalent if the Axiom of Choice (AC) is not assumed. The standard definition, namely, that a set is finite if it has $n$ elements for some natural number $n$, is absolute for transitive models of Zermelo-Fraenkel (ZF) set theory (we also call this I-finiteness, cf. Section 2, Definition 1). On the other hand, notions like Dedekind finiteness (a set is Dedekind finite if it is not equinumerous with any proper subset) are equivalent to the standard definition in every model of ZFC (ZF plus AC), but are satisfied by some infinite sets in some models of ZF.

One of the reasons that led some researchers to introduce different definitions of finiteness was a foundational concern: how to capture the concept of finiteness without making use of external objects like the natural numbers (see Tarski [at24]). That concern soon became irrelevant, as a canonical way of representing natural numbers as sets was devised; nevertheless, the definitions of finiteness remain a useful way of classifying non-well-orderable sets according to their cardinal-invariant properties.

In this paper we introduce a framework for generating definitions of finiteness systematically. We start from the following observation, which holds under AC: A

2000 Mathematics Subject Classification: 03E25, 03E20, 03E35.

Research of D. Dzhafarov and E. Hall partially supported by NSF VIGRE Grant no. DMS9983601.

The authors wish to thank Thomas Jech and Jean Rubin for helpful suggestions. 
set $X$ is finite if and only if

$$
\text { any transitive, antisymmetric binary relation on } X
$$

has a maximal element.

We modify the formula in 1.1 to obtain four definitions of finiteness by considering the following four classes of binary relations: partial orders, linear orders, tree orderings, and well orders. Notice that all these relations are transitive and antisymmetric (dropping any of these two conditions makes the notion of maximal elements useless in this context); also, reflexivity and irreflexivity are included whenever needed, since they are irrelevant for the existence of maximal elements. See Definition 4 for the actual statements (Formula 1.1 corresponds to P-finiteness).

In the last section we will look at certain "finite unions of finite sets," where finite can mean one of the less restrictive notions of finiteness mentioned above and detailed in Section 2. Note that with AC, any finite union of finite sets is finite, and so it is natural to ask which notions of finiteness are closed under "finite" unions in set theory without AC. In ZF, it is easy to verify that I-finiteness is closed under unions of I-finite families of sets, and that Dedekind finiteness is closed under unions of Dedekind-finite families, but at the same time it is known that several well studied definitions do not have this property. In this paper we will show that Ia-finite unions of Ia-finite sets (cf. Section 2, Definition 1) are P-finite (cf. Section 2, Definition 4); however, we also show that P-finiteness is not provably (in ZF) closed under P-finite unions.

\section{Definitions}

In order to establish the relative strength of the notions of finiteness we will introduce in this paper, we dedicate the first part of this section to listing some of those notions of finiteness that have already been studied in the literature in some detail. These will comprise Definitions 1,2, and 3 below, which were collected in Howard and Rubin [hr98] and De la Cruz [odlc02].

Definition 1. A set $X$ is

(1) I-finite if every non-empty family of subsets of $X$ has a maximal (or minimal) element under inclusion.

(2) Ia-finite if it is not the disjoint union of two non-I-finite sets.

(3) II-finite if every family of subsets of $X$ linearly ordered by inclusion contains a maximal element.

(4) III-finite if there is no one-to-one map from $\mathcal{P}(X)$ into a proper subset of $\mathcal{P}(X)$.

(5) IV-finite if there is no one-to-one map from $X$ into a proper subset of $X$.

(6) $\mathrm{V}$-finite if $X=\varnothing$ or there is no one-to-one map from $2 \times X$ into $X$.

(7) VI-finite if $X$ is empty or a singleton, or else there is no one-to-one map from $X \times X$ into $X$.

(8) VII-finite if $X$ is I-finite or it is not well orderable.

Definitions Ia and VII are due to Lévy, while definition IV is due to Dedekind [rd72]. Tarski [at24] introduced I, II, III and V; definition VI was attributed to Tarski by Mostowski [am38].

\section{Remark 1.}


(1) Tarski [at24] observed that a set is I-finite if and only if it is finite in the ordinary sense mentioned in the introduction (i.e., having $n$ elements with $n \in \omega)$. Thus, the terms finite and I-finite may be used interchangeably, though we shall prefer to use the former here.

(2) A set is IV-infinite if and only if it contains a well orderable infinite subset, and III-infinite if and only if it has a well orderable infinite partition.

The next group of definitions is due to Truss [jt74].

Definition 2. Define the following classes of cardinals:

(1) $\Delta_{1}=\{\mathfrak{a}: \mathfrak{a}=\mathfrak{b}+\mathfrak{c} \rightarrow \mathfrak{b}$ or $\mathfrak{c}$ is finite $\}$

(2) $\Delta_{2}=\{|X|$ : any linearly ordered partition of $X$ is finite $\}$

(3) $\Delta_{3}=\{|X|$ : any linearly ordered subset of $X$ is finite $\}$

(4) $\Delta_{4}=\left\{\mathfrak{a}\right.$ : there does not exist a function from $\mathfrak{a}$ onto $\left.\aleph_{0}\right\}$

(5) $\Delta_{5}=\{\mathfrak{a}$ : there does not exist a function from $\mathfrak{a}$ onto $\mathfrak{a}+1\}$

For $i=1, \ldots, 5$, we will say that a set $X$ is $\Delta_{i}$-finite if $|X| \in \Delta_{i}$.

Remark 2. It is clear that Ia-finiteness is equivalent to $\Delta_{1}$-finiteness. Truss notes further that II-finiteness is equivalent to $\Delta_{2}$-finiteness and III-finiteness to $\Delta_{4^{-}}$ finiteness.

Lastly, we have the following definition of Howard and Yorke [hy89]:

Definition 3. A set $X$ is $D$-finite if it is empty, a singleton, or if $X=X_{1} \cup X_{2}$, where $\left|X_{1}\right|,\left|X_{2}\right|<|X|$ (such a set is also called decomposable).

We now introduce definitions of finiteness derived from classes of order relations, as described in the introduction. It will follow from the implications proved in the next section that these are bona fide definitions of finiteness (that is, that they are equivalent to ordinary finiteness under $\mathrm{AC}$ ).

Definition 4. A set $X$ is

(1) P-finite if it contains a maximal element under every partial ordering.

(2) L-finite if it contains a maximal element under every linear ordering.

(3) W-finite if it contains a maximal element under every well ordering.

(4) Tr-finite if it contains a maximal element under every tree ordering.

Next, we introduce a means of deriving new definitions of finiteness from old ones. Let $\mathcal{Q}$ stand for any of the notions given in this section.

Definition 5. A set $X$ is

(1) $\mathcal{Q}^{1}$-finite if every set that $X$ maps onto is $\mathcal{Q}$-finite.

(2) $\mathcal{Q}^{2}$-finite if every set that maps one-to-one into $X$ is $\mathcal{Q}$-finite.

Our interest in the preceding definition will be the cases where $\mathcal{Q}$ represents one of the notions of finiteness in Definition 4.

\section{Remark 3.}

(1) It is easy to see that if $\mathcal{Q}$ is any notion of finiteness, then a set $X$ is $\mathcal{Q}^{1}$-finite if and only if every partition of $X$ is $\mathcal{Q}$-finite, and $\mathcal{Q}^{2}$-finite if and only if every subset of $X$ is $\mathcal{Q}$-finite. 
(2) The notions of finiteness in Definitions 1, 2, and 3 above are cardinal invariants (cf. De la Cruz [odlc02]). This also holds for the notions in Definition 4 , and those obtained from Definition 4 via Definition 5 , as can be easily checked case by case.

(3) Note that each of the notions given in this section yields a corresponding definition of infinity: if $\mathcal{Q}$ is any definition of finiteness, then we will say that a set is $\mathcal{Q}$-infinite if it is not $\mathcal{Q}$-finite. In the case of a I-infinite set, we will simply use the equivalent term infinite, whereas a Ia-infinite set will be called partible. An infinite, Ia-finite set is called amorphous.

\section{IMPLICATIONS BETWEEN NOTIONS}

For the sake of convenience, we will adopt the notation used by De la Cruz [odlc02]. If $\mathcal{Q}$ and $\mathcal{Q}^{\prime}$ are any notions of finiteness such that whenever a set is $\mathcal{Q}$-finite then it is $\mathcal{Q}^{\prime}$-finite, we will write $\mathcal{Q} \longrightarrow \mathcal{Q}^{\prime}$. We will write $\mathcal{Q} \longleftrightarrow \mathcal{Q}^{\prime}$ if $\mathcal{Q} \longrightarrow \mathcal{Q}^{\prime}$ and $\mathcal{Q}^{\prime} \longrightarrow \mathcal{Q}$.

Lévy [al58] established the following:

$$
\mathrm{I} \longrightarrow \mathrm{Ia} \longrightarrow \mathrm{II} \longrightarrow \mathrm{III} \longrightarrow \mathrm{IV} \longrightarrow \mathrm{V} \longrightarrow \mathrm{VI} \longrightarrow \mathrm{VII},
$$

while Truss [jt74] showed that III $\longrightarrow \Delta_{5} \longrightarrow$ IV and II $\longrightarrow \Delta_{3} \longrightarrow$ IV. Howard and Yorke [hy89] additionally showed that IV $\longrightarrow D \longrightarrow$ VII.

We begin with some lemmas which we will use to prove the main results of this section.

Lemma 1. If $\mathcal{Q}$ is any of the notions of finiteness from Section 2 , then $\mathcal{Q}^{1} \longrightarrow$ $\mathcal{Q}^{2} \longrightarrow \mathcal{Q}$.

Proof. The proof will consist mainly of reminding the reader of the definitions.

$\left(\mathcal{Q}^{1} \longrightarrow \mathcal{Q}^{2}\right)$ Given a set $X$, observe that every set that maps one-to-one into $X$ can be mapped onto by $X$. So if every set that can be mapped onto by $X$ is $\mathcal{Q}$-finite (i.e. $X$ is $\mathcal{Q}^{1}$-finite), then every set that maps one-to-one into $X$ is $\mathcal{Q}$-finite (i.e. $X$ is $\mathcal{Q}^{2}$-finite).

$\left(\mathcal{Q}^{2} \longrightarrow \mathcal{Q}\right)$ Now let $X$ be $\mathcal{Q}^{2}$-finite. That means that every set that maps one-to-one into $X$, including $X$ itself, is $\mathcal{Q}$-finite.

It is easy to check now that the notions $\mathcal{Q}^{2}$ and $\left(\mathcal{Q}^{2}\right)^{2}$ are equivalent, as are the notions $\mathcal{Q}^{1},\left(\mathcal{Q}^{1}\right)^{1},\left(\mathcal{Q}^{1}\right)^{2}$, and $\left(\mathcal{Q}^{2}\right)^{1}$, meaning that Definition 5 yields at most two new notions of finiteness for each notion $\mathcal{Q}$.

The task for the remainder of this section will be to find all relations of implication between each of the new notions P, L, Tr, and W, as well as their Definition 5 variants, and the old notions from Definitions 1-3. Several of the old notions will be shown equivalent to new ones, so that what we have are new characterizations of old notions of finiteness in terms of order relations. Other notions from Definition 4 really are new. The implications proved in this section will be the strongest possible in $\mathrm{ZF}$ between these notions; the independence results in the next section will show that no stronger implications are provable in ZF.

Remark 4. Several implications between the new notions are clear. We immediately have $\mathrm{P} \longrightarrow \mathrm{L} \longrightarrow \mathrm{W}$ and $\mathrm{P} \longrightarrow \mathrm{Tr} \longrightarrow \mathrm{W}$ (since every linear order is a partial order, and so on). More implications follow from Lemma 1 above, and from the fact that whenever $\mathcal{Q}$ and $\mathcal{R}$ are two notions with $\mathcal{Q} \longrightarrow \mathcal{R}$, we have $\mathcal{Q}^{1} \longrightarrow \mathcal{R}^{1}$ and $\mathcal{Q}^{2} \longrightarrow \mathcal{R}^{2}$. 
We now study each of the four notions $\mathrm{P}, \mathrm{L}, \mathrm{Tr}$, and $\mathrm{W}$ one by one together with their Definition 5 variants, beginning with P-finiteness.

Theorem 1. $\mathrm{P}^{1} \longleftrightarrow \mathrm{P}^{2} \longleftrightarrow \mathrm{P}$

Proof. In view of Lemma 1 , it suffices to show that $\mathrm{P} \longrightarrow \mathrm{P}^{1}$. Take $X$ to be a P-finite set, and suppose $\langle S, \leq\rangle$ is any partial order such that $X$ maps onto $S$. Say $f: X \rightarrow S$ is a surjection. We define a relation $\preceq$ on $X$ by the rule that, for all $x, y \in X, x \preceq y$ if and only if either $x=y$ or else $f(x) \neq f(y)$ and $f(x) \leq f(y)$. It is easy to see that $\preceq$ partially orders $X$, and consequently that there is an $x \in X$ which is maximal in $X$ under $\preceq$. Now suppose there exists an $s \in S$ such that $f(x) \leq s$, meaning there must be a $y \in X$ such that $f(y)=s$ and $x \preceq y$. But this only happens if $x=y$, so $s=f(x)$, and $f(x)$ is in fact maximal in $S$ under $\leq$. Therefore, we see that $S$ is P-finite and, as a result, that $X$ is $\mathrm{P}^{1}$-finite.

The next theorem shows that $\mathrm{P}$-finiteness is equivalent to a modification of $\mathrm{P}$ finiteness that may look stronger at first glance. Given a partial order $\preceq$ on a set $X$, a $\preceq$-chain is a subset of $X$ that is linearly ordered by $\preceq$. It is easy to see that $X$ being P-finite is equivalent to $X$ having no infinite chains for any partial order $\preceq$. It is less obvious that for each $\preceq$ on $X$, the size of the $\preceq$-chains must be bounded.

Theorem 2. A set $X$ is $\mathrm{P}$-finite if and only if for each partial order $\preceq$ on $X$, there is a positive integer $M$ such that every $\preceq$-chain of $X$ has length less than $M$.

Proof. Let $X$ be a P-finite set and fix a partial order $\preceq$ on $X$. For $x \in X$, let $f(x)$ be the maximum integer $n$ such that there is a chain of length $n$ with $x$ at the bottom, if such a maximum exists. For example, if $x$ is a maximal element, then $f(x)=1$. First, observe that the range of $f$ must be a finite subset of $\omega$ (since $X$ is $\mathrm{P}^{1}$-finite). Let $M$ be the maximum of the range of $f$. It just remains to show that $f(x)$ is defined for all $x \in X$; it follows that $X$ has no $\preceq$-chain of length greater than $M$.

Let $Y$ be the subset of $X$ on which $f$ is undefined, and suppose by way of contradiction that $Y$ is non-empty. Since $X$ is $\mathrm{P}^{2}$-finite, $Y$ is $\mathrm{P}$-finite and must have a $\preceq$-maximal element $y$. Since $f(y)$ is undefined, there must be a chain $C \subset X$ starting with $y$ and having length at least $M+2$. Let $x$ be the second element in the chain, so that $C \backslash\{y\}$ is a chain starting with $x$ of length at least $M+1$. But now we have a contradiction because $C \backslash\{y\}$ has no elements in $Y$ (since $y$ is maximal in $Y$ ), and so $f(x)$ must be defined and at most $M$.

The "moreover" part of the next theorem will be used in Section 5 .

Theorem 3. Ia $\longrightarrow \mathrm{P} \longrightarrow$ II. Moreover, any $\mathrm{P}-$ infinite set has a partition into two P-infinite sets.

Proof. To see that $\mathrm{P} \longrightarrow \mathrm{II}$ is straightforward, since this implication may be rewritten as $\mathrm{P}^{1} \longrightarrow \mathrm{L}^{1}$ (Theorems 1 and 5 ), and $\mathrm{P}^{1} \longrightarrow \mathrm{L}^{1}$ follows from $\mathrm{P} \longrightarrow \mathrm{L}$.

Now let $X$ be any P-infinite set. To prove Ia $\longrightarrow \mathrm{P}$ we just need to show that $X$ has a partition into two infinite parts. It is barely any more work to show that $X$ has a partition into two P-infinite parts, and that is what we will do.

There exists a partial ordering $\preceq$ on $X$ under which $X$ contains no maximal element. For each $x \in X$ define $B_{x}=\{y \in X: y \preceq x\}$. In case there is an $x \in X$ such that $B_{x}$ is P-infinite, then we are done since the complement of $B_{x}$ in $X$ clearly has no $\preceq$-maximal element so is also P-infinite. 
So suppose $B_{x}$ is P-finite for each $x \in X$. In this case we can define a rank function on $X$. Let $r(x)$ be the maximum length of any $\preceq$-chain in $B_{x}$. Theorem 2 shows that this $r$ is defined for each $x \in X$. Since $X$ has no $\preceq$-maximal element, the range $\operatorname{Ran}(r)$ of this rank function must be an unbounded subset of $\omega$. Partition $\operatorname{Ran}(r)$ into two infinite parts. Then the inverse image of each part is a subset of $X$ that $r$ maps onto an infinite well orderable set, so must be P-infinite (in fact III-infinite).

Next we study L-finiteness. It should not be surprising that $\Delta_{2}$ and $\Delta_{3}$ finiteness have characterizations in terms of L-finiteness, since their original definitions are in terms of linear orderings.

Lemma 2. If $X$ is infinite and $\langle X, \preceq\rangle$ is a linear order, there exists $Y \subset X$ such that either $\langle Y, \preceq\rangle$ or $\langle Y, \succeq\rangle$ (where $\succeq=\{(a, b):(b, a) \in \preceq\}$ ) has no maximum element.

Proof (by contrapositive). Assume both that (1) every subset of $X$ has a $\preceq$-maximum and (2) every subset of $X$ has a $\preceq$-minimum (or, equivalently, a $\succeq$-maximum). By (2), we have that $X$ is well ordered by $\preceq$, say in type $\alpha$. Now any ordinal of type $\geq \omega$ has an infinite subset with no maximal element (e.g., the set $\omega$ ). So by (1), we know that $\alpha<\omega$, and consequently $X$ is finite.

Lemma 3. A set $X$ is L-finite if and only if it is finite or else not linearly orderable.

Proof. If $X$ is finite or has no linear ordering, then $X$ clearly satisfies the definition of L-finiteness. Conversely, suppose $X$ is infinite and has a linear ordering $\leq$. By Lemma 2, there is a subset $Y \subseteq X$ with no $\leq$-maximum (or minimum, but in that case simply reverse the order of $\leq$ ). Now it is easy to form a new linear order $\preceq$ on $X$ by modifying $\leq$ so that the two orders agree on $Y$ and on $X \backslash Y$, but in which all elements of $X \backslash Y$ precede all elements of $Y$ under $\preceq$. This shows $X$ to be L-infinite, since it has no $\preceq$-maximum.

Theorem 4. $\Delta_{3} \longleftrightarrow \mathrm{L}^{2}$

Proof. A set $X$ is $\mathrm{L}^{2}$-finite if and only if every subset of $X$ is L-finite (Remark 3.1). This is equivalent (by Lemma 3 ) to the condition that every linearly orderable subset of $X$ is finite; i.e., $X$ is $\Delta_{3}$-finite.

Theorem 5. II $\longleftrightarrow \mathrm{L}^{1}$

Proof. A set $X$ is II-finite only if it is $\Delta_{2}$-finite (see Remark 2), which means that every linearly ordered partition of $X$ is finite. The proof of this theorem now proceeds just as the proof of Theorem 4, but referring to partitions of $X$ instead of subsets of $X$.

In contrast to $\mathrm{L}^{1}$ and $\mathrm{L}^{2}$, L-finiteness itself is not equivalent to any of our other notions of finiteness. The strongest implications provable in $\mathrm{ZF}$ are just the obvious ones in the following statement.

Theorem 6. $\Delta_{3} \longrightarrow \mathrm{L} \longrightarrow \mathrm{VII}$

Proof. Straightforward; rewrite as $\mathrm{L}^{2} \longrightarrow \mathrm{L} \longrightarrow \mathrm{W}$ (by Theorems 4 and 7 ).

The following equivalences involving $\mathrm{W}$-finiteness are closely analogous to equivalences above involving L-finiteness. 
Theorem 7. VII $\longleftrightarrow \mathrm{W}$

Proof. Analogously to the proof of Lemma 3, the proof of VII $\longrightarrow \mathrm{W}$ is trivial. Conversely, suppose $X$ is not VII-finite, so it is both infinite and well-orderable. In that case, the cardinality of $X$ is some $\aleph$, say $\aleph_{\alpha}$. But then $X$ has a well-ordering whose type is the initial ordinal $\aleph_{\alpha}$ (or $\omega_{\alpha}$ if you prefer that notation for ordinals). Since $\aleph_{\alpha}$ has no maximal element, $X$ is not W-finite.

Theorem 8. IV $\mathrm{W}^{2}$

Proof. A set $X$ is $\mathrm{W}^{2}$-finite if and only if every subset of $X$ is W-finite (Remark 3.1). This is equivalent (by Theorem 7 ) to the condition that every well-orderable subset of $X$ is finite; i.e., $X$ is IV-finite.

Theorem 9. $\mathrm{W}^{1} \longleftrightarrow$ III

Proof. It is probably simplest to replace W by VII and III by $\Delta_{4}$ and observe that VII $^{1}$ ("Every set that $X$ maps onto is finite or non-well-orderable") is equivalent to $\Delta_{4}$ (" $X$ does not map onto $\aleph_{0} "$ ").

Remark 5. Several of the notions of finiteness in this paper fall between $\mathrm{W}^{1}$ and W (i.e. between III and VII), as can be seen in Figure 1 at the end of this section. For any such notion $\mathcal{Q}$ with $\mathrm{W}^{1} \longrightarrow \mathcal{Q} \longrightarrow \mathrm{W}$, we have $\mathcal{Q}^{1} \longleftrightarrow \mathrm{W}^{1} \longleftrightarrow$ III. (Recall $\left(\mathrm{W}^{1}\right)^{1} \longleftrightarrow \mathrm{W}^{1}$ and Remark 4.)

Finally we study Tr-finiteness.

Theorem 10. $\Delta_{5} \longleftrightarrow \operatorname{Tr}^{2}$

Proof. $\left(\Delta_{5} \longrightarrow \operatorname{Tr}^{2}\right)$ Let $X$ be $\operatorname{Tr}^{2}$-infinite, so that there is a subset $S$ of $X$ with a tree ordering $\preceq$ that has no maximal element. Fix some $u \notin X$, and let $g$ be the function that is the identity on $X \backslash S$ and defined on $S$ as follows:

$$
g(s)=\left\{\begin{array}{l}
\text { the } \preceq \text {-predecessor of } s, \text { if such a predecessor exists } \\
u, \text { if } s \text { has no } \preceq \text {-predecessor }
\end{array} .\right.
$$

Then $g$ is a surjection from $X$ onto $X \cup\{u\}$, proving that $X$ is $\Delta_{5}$-infinite.

$\left(\operatorname{Tr}^{2} \longrightarrow \Delta_{5}\right)$ Suppose now that $X$ is $\Delta_{5}$-infinite, that is, that there is a function $f$ from $X$ onto $X \cup\{u\}$, with $u \notin X$. We define a tree ordering $\preceq$ of height $\omega$ without maximal elements on a subset of $X$ by recursion: take $T_{0}=f^{-1}(u)$ (the elements here are the "roots" of the tree); if levels $T_{0}, \ldots, T_{n}$ have been defined, as well as $\preceq$ on $T_{0} \cup \cdots \cup T_{n}$, we define $T_{n+1}=f^{-1}\left(T_{n}\right)$ and for each $a \in T_{n+1}$ we set $b \preceq a$ iff $b \in T_{0} \cup \cdots \cup T_{n}$ and $b=f(a)$ or $b \preceq f(a)$ (or if $b=a$ ). It can be easily checked that $\preceq$ is a tree ordering on $T=\bigcup_{n \in \omega} T_{n}$ that satisfies the required properties.

Theorem 11. $\Delta_{5} \longrightarrow \operatorname{Tr} \longrightarrow \mathrm{V}$

Proof. The first implication is clear since $\Delta_{5} \longleftrightarrow \operatorname{Tr}^{2}$ (Theorem 10).

To prove $\operatorname{Tr} \longrightarrow \mathrm{V}$, suppose that $X$ is $\mathrm{V}$-infinite, so that there exists a bijection $F: 2 \times X \rightarrow X$. The function $f: X \rightarrow X$ given by $f(x)=F(\langle 0, x\rangle)$ is one-to-one; let $Y=f^{\prime \prime} X$ and $Z=X \backslash Y$. For each $z \in Z$ we have that $f(z) \neq z$, so the orbit $\left\{f^{n}(z): n \in \omega\right\}$ is infinite and can be naturally ordered in order type $\omega$.

The union of all the $f$-orbits of elements of $Z$ is then naturally ordered as a tree of height $\omega$ with no maximal elements (each $z \in Z$ is a root and there is no 


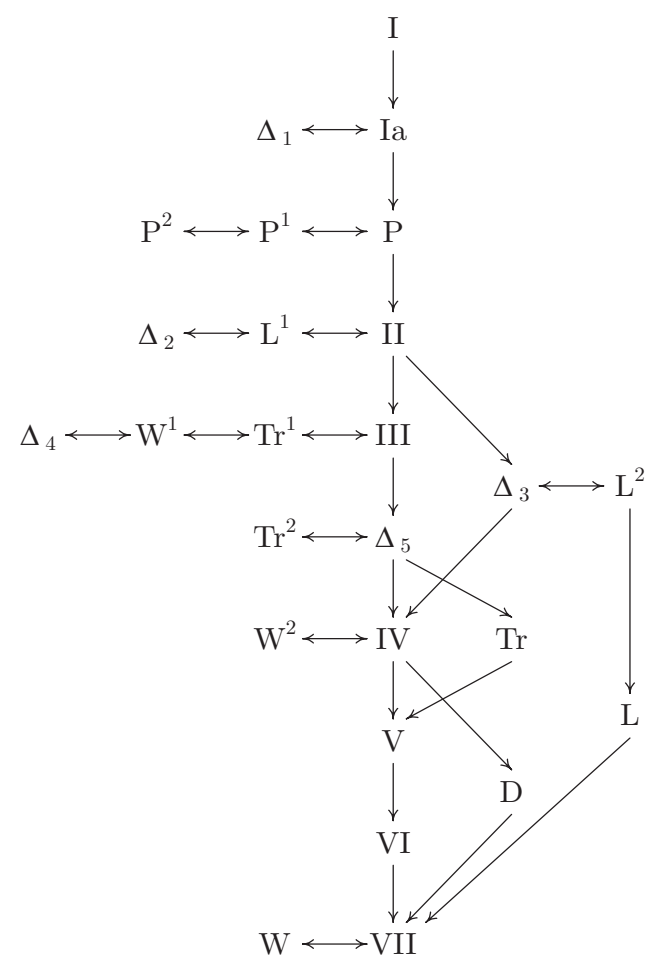

Figure 1. Relations between definitions of finiteness. Arrows indicate implications (cf. Section 3). Arrows pointing in only one direction are not reversible in ZF (cf. Section 4).

branching). If there are elements $y \in Y$ that are not in that union we place them below the root

$$
F\left(1, f^{-1}(y)\right)
$$

This finishes defining the desired tree order on all of $X$.

(Notice that a small variation of the proof above yields the ZF fact that if $|X|=2 \cdot|X|$ then $|X|=\aleph_{0} \cdot|X|$.)

Corollary 1. $\operatorname{Tr}^{1} \longleftrightarrow \mathrm{W}^{1} \longleftrightarrow \mathrm{III}$

Proof. See Remark 5; Theorem 11 puts the notion Tr between III and VII.

All the implications between notions of finiteness are summarized in Figure 1. Notice that if $\mathcal{Q}$ is any notion of finiteness given in Section 2 , then I $\longrightarrow \mathcal{Q} \longrightarrow$ VII. Now if the Axiom of Choice is assumed, and every set is well orderable, then VII $\longrightarrow$ I. Thus all the notions in Figure 1 are legitimate notions of finiteness in the sense of being equivalent to I-finiteness under AC.

\section{INDEPENDENCE RESULTS}

In this section, we show that the implications established above are the only ones that can be proved in ZF. 
We begin with references to the known results. Lévy [al58] proved that if $\mathcal{Q}$ and $\mathcal{Q}^{\prime}$ are any of the notions listed in Definition 1 , then $\mathcal{Q} \nrightarrow \mathcal{Q}^{\prime}$ whenever $\mathcal{Q}$ is below $\mathcal{Q}^{\prime}$ in that list. (The techniques to give these independence results in ZF were not available to Lévy in 1958. He worked in the context of the weaker theory ZFA, but his results all transfer to ZF by the Jech-Sochor Embedding Theorem; see comments on this below.) Truss [jt74] showed III $\nrightarrow \Delta_{3}$ and $\Delta_{3} \nrightarrow \Delta_{5}$, meaning that for $\mathcal{Q}$ in Definition $1, \mathcal{Q} \longrightarrow \Delta_{3}$ only if $\mathcal{Q}$ is listed above III, and that $\Delta_{3} \longrightarrow \mathcal{Q}$ only if $\mathcal{Q}$ is IV or listed below IV. It also follows, since $\Delta_{3} \longrightarrow \mathrm{IV}$, that $\mathrm{IV} \nrightarrow \Delta_{5}$. De la Cruz [odlc02] established that $\Delta_{5} \nrightarrow \mathrm{III}$ and that V $\nrightarrow D$, while Howard and Yorke [hy89] showed that $D \nrightarrow$ VI. So $\mathcal{Q} \longrightarrow D$ only if $\mathcal{Q}$ is above $\mathrm{V}$ in Definition 1 , and $D \nrightarrow \mathcal{Q}$ for any $\mathcal{Q}$ in Definition 1 above VII. This settles all questions about implications between the notions of finiteness from Definitions 1, 2, and 3 of Section 2.

We now begin to address the implications involving notions from Definition 4 and Definition 5. We can use the known fact that $\mathrm{I} \nrightarrow$ Ia to separate P-finiteness from Ia-finiteness. Recall that an amorphous set is one that is Ia-finite but infinite (i.e., an infinite set with no partition into two infinite parts).

Theorem 12. $\mathrm{P} \nrightarrow \mathrm{Ia}$

Proof. Working from ZF plus "There exists an amorphous set" (which is equiconsistent with ZF by remarks above) we prove that there is a set which is P-finite and Ia-infinite. Given an amorphous set $X$, there must be another amorphous set $Y$ with $X \cap Y=\varnothing$. Clearly $X \cup Y$ is not amorphous; it is Ia-infinite.

Both $X$ and $Y$ are Ia-finite and hence P-finite (Ia $\longrightarrow \mathrm{P}$ by Theorem 3). Any partial order on $X \cup Y$ must have an element $a$ which is maximal in $X$ and an element $b$ which is maximal in $Y$. At least one of $a$ or $b$ must be maximal in $X \cup Y$, which shows that $X \cup Y$ is $\mathrm{P}$-finite.

For most of the remaining independence results, we will work directly with models of set theory rather than relying on previously obtained independence results as in the proof of Theorem 12. We will obtain our results in the theory ZFA, a weakened version of ZF which allows for the existence of a atoms, objects with no elements yet different from each other and from the empty set (extensionality must be weakened so that it applies only to sets); we will assume that the class $A$ of atoms is actually a set. Models of ZF are rigid, but in a model $M$ of ZFA the permutations of $A$ correspond exactly with the automorphisms of the universe (that is, $\in$-automorphisms of $M$ which constitute a definable class in $M$ ). When $M$ satisfies ZFA $+\mathrm{AC}+$ " $A$ is infinite" (a theory that is consistent relative to $\mathrm{ZF}$ ), the rich provision of automorphisms allows us to construct so-called permutation submodels of $M$, which are models of ZFA where AC fails in a controlled way.

Even though ZFA is a weaker theory, the independence results we obtain in this way will also hold in ZF, by virtue of the Jech-Sochor Embedding Theorem [tjj73, Chapter 6, js66], which allows for the embedding of initial segments of permutation models into models of ZF. Without going into the details, we only remark here that if a sentence of the form

$$
\exists x\left(x \text { is } \mathcal{Q} \text {-finite and } \mathcal{Q}^{\prime}\right. \text {-infinite) }
$$

is true, then its truth is realized in an initial segment of the universe.

We give a summary of the construction of permutation models. For a detailed description of permutation models, see [tjj73]; we will make note below where our 
formulation differs slightly from the one there. Fix a model $V$ of ZFA $+\mathrm{AC}+$ " $A$ is infinite", where $A$ is the set of atoms of $V$, and a group $G \in V$ of permutations of $A$. Abusing the notation, we identify each $\pi \in G$ with the class function $\pi: V \rightarrow$ $V$ defined by the rule $\pi(\varnothing)=\varnothing$ and $\pi(x)=\{\pi(y): y \in x\}$ for all $x$. For any $x \in V$, we call the stabilizer of $x$ in $G$ the group $G_{x}=\{\pi \in G: \pi x=x\}$; the pointwise stabilizer of $x$ in $G$ is the group $\operatorname{fix}_{G}(x)=\left\{\pi \in G: \pi\lceil x=\operatorname{Id}\}=\bigcap_{y \in x} G_{y}\right.$. Now, if $x$ and $y$ are objects (sets or atoms), we say $x$ supports $y$ if $\operatorname{fix}_{G}(y) \subseteq G_{y}$. We define a base of supports (for $G$ ) as a set $B$ satisfying the following properties: $G_{B}=G$; every $a \in A$ is supported by some $b \in B$; for every $b_{0}, b_{1} \in B$, there is a $c \in B$ which supports every element of $b_{0} \cup b_{1}$. (In [tjj73], a permutation model is defined in terms of a normal filter on $G$ instead of a base of supports for $G$. The formulations are equivalent. The filter associated with the base of supports $B$ is the filter generated by $\left\{\operatorname{fix}_{G}(b): b \in B\right\}$.) A commonly used base of supports is the set of finite subsets of $A$, called the base of finite supports. Now we say $x \in V$ is symmetric (with respect to $B$ and $G$ ), if it is supported by some $b \in B$; we say it is hereditarily symmetric if every element of the transitive closure of $x$ is symmetric. The class $\mathcal{N}_{G, B}$ of all hereditarily symmetric sets with respect to $G$ and $B$ is a model of ZFA, and we call it the permutation model obtained from $G$ and $B$.

Howard and Yorke [hy89] have presented the permutation model M6, whose construction is given below, and proved that in this model, the set $A$ is $D$-infinite.

Theorem 13. In the model M6, the set $A$ of atoms is Tr-finite and L-finite.

Proof. The model $M 6$ is constructed by first taking a model of ZFA+AC and letting $A$ be ordered in the order type of the rationals. Then $M 6$ is the permutation model obtained from the group $G$ of permutations which move finitely many elements of $A$ and the base of supports $B$ of bounded subsets of $A$.

Let $\preceq$ be a tree ordering on $A$ that is in $M 6$; we will find a $\preceq$-maximal element. Since $\preceq$ is symmetric, there is a support $E \in B$ such that fix $(E)$ is a subset of $G_{\preceq}$; in other words, every $\pi \in \operatorname{fix}(E)$ is a $\preceq$-automorphisms of $A$. Pick two distinct elements $a$ and $b$ in $A \backslash E$. The permutation $\pi:=(a, b)$ which just switches $a$ and $b$ is in $\operatorname{fix}(E)$. It follows that $a \preceq b$ iff $b \preceq a$; these cannot be both true so $a$ and $b$ are incomparable under $\preceq$. If $a$ is not a maximal element, then there is $c \in A$ such that $a \preceq c$ and $b \preceq c$. But then $\pi a \preceq \pi c$, which means $b \preceq c$ and we have a contradiction. So $a$ is a maximal element.

Next, suppose $\leq$ is a linear ordering of $A$ in $M 6$ and let $E \in B$ be a support for $\leq$. Pick two distinct elements $a$ and $b$ in $A \backslash E$. Arguing as above we find that $a$ and $b$ must be incomparable under $\leq$, which is a contradiction since $\leq$ is a linear order. Hence, there is no linear ordering of $A$ at all in $M 6$.

Corollary 2. $\operatorname{Tr} \nrightarrow D, \mathrm{~L} \nrightarrow D, \operatorname{Tr} \nrightarrow \mathrm{IV}$, and $\mathrm{L} \nrightarrow \mathrm{IV}$.

Proof. It was just shown that in the permutation model $M 6$, the set $A$ of atoms is Tr-finite and L-finite. That $A$ is D-infinite is in [hy89]. To see that $A$ is IV-infinite in $M 6$, take any infinite $E \in B$. Since each $E \in B$ is subset of $A$ and a support for a well-ordering of itself, $A$ has well-ordered infinite subsets in $M 6$.

Consequently, $\operatorname{Tr} \longrightarrow \mathcal{Q}$ only if $\mathcal{Q}$ is V, VI, or VII, or equivalent to one of these.

Any union of an amorphous set with a countably infinite set is another, perhaps simpler example of a set that is Tr- and L-finite but IV-infinite. However, the example of Corollary 2 may be preferable in that it shows Tr- and L-finiteness of 
a set $X$ to be consistent with the stronger property "Every infinite subset of $X$ is IV-infinite."

Theorem 14. $\Delta_{3} \nrightarrow \operatorname{Tr}$

Proof. Consider Fraenkel's Second Model, in which $A$ is the union of a disjoint family of countably many pairs, $A=\bigcup\left\{P_{n}: n \in \omega\right\}$, with no choice function.

For every $n \in \omega$, define $F_{n}$ as a the set of all choice functions on $\left\{P_{k}: k \leq n\right\}$ and set $F=\bigcup\left\{F_{n}: n \in \omega\right\}$. It is shown in Truss [jt74] that $F$ is $\Delta_{3}$-finite in this model. However, the $\subseteq$ relation on $F$ is a tree ordering with no maximal element, so $F$ is Tr-infinite.

Since $\Delta_{3} \longrightarrow$ IV, it follows that $\mathcal{Q} \nrightarrow$ Tr for any $\mathcal{Q}$ listed below III in Definition 1 . We also have that $\Delta_{5} \longrightarrow \operatorname{Tr}$ but $\Delta_{5} \nrightarrow \Delta_{3}$, meaning $\operatorname{Tr} \nrightarrow \Delta_{3}$.

Theorem 15. III $\nrightarrow \mathrm{L}$

Proof. Truss [jt74] showed that the set $A$ in Mostowski's ordered model is III-finite. This set is infinite and linearly orderable (see Howard and Rubin [hr98]) and so Linfinite.

It follows from the previous theorem, since III $\longrightarrow$ IV $\longrightarrow D$ and III $\longrightarrow \Delta_{5} \longrightarrow$ $\operatorname{Tr}$ that $\mathcal{Q} \nrightarrow \mathrm{L}$ for any $\mathcal{Q}$ in Definition 1 below II, and also that $D \nrightarrow \mathrm{L}$ and $\operatorname{Tr} \nrightarrow \mathrm{L}$.

The last task of this section is to show that II $\nrightarrow \mathrm{P}$. For the remainder of this section, the relation symbol $\preceq$ will refer to a universal homogeneous partial order on $A$, where $A$ is a set of atoms. A partial order is called universal if every finite partial order can be embedded in it, and is called homogeneous if every order isomorphism between two of its finite subsets can be extended to an automorphism of the entire set. Notice that such a partial order has no maximal element. For a proof of the existence of a countable universal homogeneous partial order, see Jech [tjj73].

Given a finite subset $E$ of $A$, we say that two elements $a \in A$ and $b \in A$ have the same $E$-type iff there is a $\preceq$-isomorphism from $E \cup\{a\}$ to $E \cup\{b\}$ whose restriction to $E$ is the identity. (Equivalently, $b$ and $c$ have the same $E$-type iff $b$ and $c$ satisfy exactly the same sentences in the structure $\left\langle A, \preceq, e_{1}, \ldots, e_{n}\right\rangle$.)

For a proof of part 1 of the following lemma, which is straightforward, see [tjj73]. Part 1 can be used to prove part 2 (which is not used in full until Theorem 19).

Lemma 4. Let $\langle A, \preceq\rangle$ be a countable universal homogeneous poset.

(1) Let $\langle F, \leq\rangle$ be a finite poset. If $E \subset F$ and $f: E \rightarrow A$ is a partial order embedding, then there is an embedding of $F$ into $A$ that extends $f$.

(2) Let $E$ be a finite subset of $A$; let $a$ and $b$ be $\preceq$-incomparable elements of $A$ with the same $E$-type, and let $D$ be a finite subset of $A$ disjoint from $E \cup$ $\{a, b\}$. Then there is an automorphism $\pi$ of $\langle A, \preceq\rangle$ that fixes $E$ pointwise, switches $a$ and $b$, and such that $\pi D \cap D=\varnothing$.

Theorem 16. II $\nrightarrow \mathrm{P}$

Proof. Let $\mathcal{N}$ be the permutation model obtained from the group $G$ of all order automorphisms of $\langle A, \preceq\rangle$, where $\preceq$ is a countable universal homogeneous partial order, and from the base $B$ of finite supports. Since $\preceq$ is symmetric and has no maximal element, $A$ is L-infinite in $\mathcal{N}$. 
Recall that II $\longleftrightarrow \mathrm{L}^{1}$ (Theorem 5). We will show that $A$ is $\mathrm{L}^{1}$-finite in $\mathcal{N}$ : Every linearly ordered set mapped onto by $A$ is finite. Let $\langle T, \leq\rangle$ be a set with a linear order in $\mathcal{N}$, and also in $\mathcal{N}$ let $t: A \rightarrow T$ be an onto function. Let $E \in B$ be a support for both $t$ and $\leq$.

Let $a$ and $b$ be any two elements of $A$ that have the same $E$-type; we will show that $t(a)=t(b)$. First, suppose $a$ and $b$ are incompatible under $\preceq$. Then there is a $\pi \in G$ that switches $a$ and $b$ while fixing $E$ pointwise. Suppose without loss of generality that $t(a) \leq t(b)$. Since $\pi$ preserves both $t$ and $\leq$, we have $t(\pi a) \leq t(\pi b)$; in other words $t(b) \leq t(a)$. Thus $t(a)=t(b)$. More generally, $a$ and $b$ may not be incompatible. However, by Lemma 4 there is a $c \in A$ such that $c$ has the same $E$-type as $a$ and $b$ and such that $c$ is compatible with neither $a$ nor $b$, in which case the argument just presented shows that $t(a)=t(c)=t(b)$. Now, since there are only finitely many $E$-types, the range $T$ of $t$ must be finite, and this finishes the proof that $A$ is $\mathrm{L}^{1}$-finite in $\mathcal{N}$.

\section{Closure under Unions}

For a class $\mathcal{C}$ of sets, we say that $\mathcal{C}$ is closed under unions iff whenever both $X \in \mathcal{C}$ and $X \subset \mathcal{C}$ we have $\bigcup X \in \mathcal{C}$. We will use the terminology " $\mathcal{Q}$ "-finiteness is closed under unions" as shorthand for "The class of $\mathcal{Q}$-finite sets is provably closed under unions, in ZF." Since the class of finite sets is closed under unions, closure under unions is a reasonable property for a notion of finiteness to have. We will investigate closure under unions for some of the strongest notions of finiteness defined in Section 1.

The class of II-finite sets is closed under unions. (Recall that II $\longleftrightarrow \mathrm{L}^{1}$. It is easy to check that both (1) L-finiteness is closed under unions and (2) $\mathcal{Q}^{1}$-finiteness is closed under unions whenever $\mathcal{Q}$-finiteness is.) However, this is the strongest notion of finiteness we have studied which has this property. The class of Ia-finite sets famously lacks closure under unions; a disjoint union of two amorphous sets is obviously not amorphous. A Ia-finite union of Ia-finite sets must be II-finite, since II-finiteness is closed under unions; we will show that it must in fact be Pfinite, giving a stronger closure property (recall that P-finiteness is intermediate in strength between Ia- and II-finiteness). We will also show that P-finiteness itself is not closed under unions, so the best we can currently say about a P-finite union of P-finite sets is that it is II-finite.

We omit the proof of the following simple characterization.

Lemma 5. A given set $X$ is a finite union of Ia-finite sets if and only if there is some $n \in \omega$ such that every partition of $X$ has at most $n$ infinite parts.

Next, we show P-finiteness for a limited class of Ia-finite unions.

Lemma 6. Any Ia-finite union of finite sets is also a finite union of Ia-finite sets (and hence is P-finite).

Proof. Let $S$ be a Ia-finite collection of finite sets. Since $S$ is Ia-finite, all but finitely many of its members must have the same size. Set aside the finitely many oddballs and assume that all members of $S$ have cardinality $n$. Let $X=\bigcup S$.

Suppose, by way of contradiction, that $X$ has a partition $T$ into $n+1$ infinite sets. For each $s \in S$, let $f(s)$ be the set of elements of $T$ that have empty intersection with $s$. Note that $f(s)$ is non-empty for each $s$, since $|s|<|T|$. Now, since $S$ is 
Ia-finite, there is a $T^{\prime} \subset T$ such that $f(s)=T^{\prime}$ for all but finitely many $s \in S$. But then $t \in T^{\prime}$ has non-empty intersection with only finitely many $s \in S$, so $t$ is finite, and we have a contradiction.

Thus $X$ has no partition into more than $n$ infinite sets, and the proof is finished by Lemma 5. (Finally, note that a finite union of Ia-finite sets is easily seen to be P-finite, as in the proof of Theorem 12.)

Now we will show that every Ia-finite union of Ia-finite sets is P-finite. We remark that the idea of the following proof can be expressed in terms of ultrafilters. Every amorphous set has a natural non-trivial ultrafilter (comprising the cofinite subsets). Although the empty ultrafilter is usually disallowed, take it to be the natural ultrafilter on any finite set. This gives a natural ultrafilter (which may be empty) on any Ia-finite union of Ia-finite sets. Expressed in these terms, the previous lemma says that a set which is small with respect to this ultrafilter must be P-finite; this is exploited in the next proof.

Theorem 17. A Ia-finite union of Ia-finite sets is $\mathrm{P}$-finite.

Proof. Let $S$ be a Ia-finite set of Ia-finite sets, and let $x=\bigcup S$. To show that $x$ is P-finite, it suffices to show, by Theorem 3 , that $x$ cannot be partitioned into two P-infinite parts.

Let $\{a, b\}$ be any partition of $x$ into two parts. Since each $t \in S$ is Ia-finite, either $t \cap a$ or $t \cap b$ is finite. Thus the two subsets of $S$ defined here are disjoint:

$$
\begin{aligned}
& S_{a}=\{t \in S: t \cap a \text { is infinite }\} \\
& S_{b}=\{t \in S: t \cap b \text { is infinite }\} .
\end{aligned}
$$

Since $S$ is Ia-finite, one of those two sets, say $S_{a}$, is finite. Think of $a$ as the union of the two sets $\bigcup\left\{t \cap a: t \in S_{a}\right\}$ and $\bigcup\left\{t \cap a: t \in S \backslash S_{a}\right\}$. The former is a finite union of Ia-finite sets, and the latter is a Ia-finite union of finite sets. By Lemma 6 , $a$ is P-finite.

As we show next, the converse of Theorem 17 cannot be proved without assuming some choice.

Theorem 18. It is consistent in the theory ZF that not every $\mathrm{P}$-finite set is a Ia-finite union of Ia-finite sets.

Proof. Let $\mathcal{N}$ be the permutation model obtained from the group $G$ of all permutations of an infinite set $A$ of atoms, and the base $B$ of supports given by finite partitions of $A$.

To see that $A$ is P-finite, let $\leq$ be a partial order of $A$ in $\mathcal{N}$, supported by a finite partition $E$ of $A$. Let $n=|E|$. Consider a chain of elements $a_{0} \leq \cdots \leq a_{n}$. There must be $i$ and $j$ in $n$ such that $a_{i}$ and $a_{j}$ are both in the same member of $E$. Then the permutation $\pi=\left(a_{i}, a_{j}\right)$ is in $\operatorname{fix}(E)$, and it follows that $a_{i} \leq a_{j}$ iff $a_{j} \leq a_{i}$, so in fact $a_{i}=a_{j}$. This shows that all $\leq$-chains have size at most $n$, and consequently there must be a $\leq$-maximal element.

Now suppose, working in $\mathcal{N}$, that $T$ is a Ia-finite set of Ia-finite subsets of $A$; we will show that $\bigcup T \neq A$. First observe that since each $E \in B$ is a member of $\mathcal{N}$, there is no subset of $A$ that is amorphous in $\mathcal{N}$. Thus the elements of $T$ are in fact finite subsets of $A$. By Lemma 6 , there is a finite set $S$ of Ia-finite sets such that $\bigcup T=\bigcup S$. Again, the Ia-finite members of $S$ must be finite, and so $\bigcup S$ is finite and therefore just a proper subset of $A$. 
If it were true that P-finiteness were closed under union, then Theorem 17 could have been obtained as a trivial consequence. However, the following theorem shows that P-finiteness is not closed under unions. The proof uses forcing to extend a permutation model. Forcing in the context of ZFA is not much different from ZF forcing, once it is understood that atoms are to be names for themselves (like the empty set is a name for itself when forcing in ZF). Details may be found in [bs89].

Theorem 19. It is consistent with ZF for a P-finite union of P-finite sets not to be P-finite.

Proof. Let $\mathcal{N}$ be the finite support permutation model used in Theorem 16 to show II $\leftrightharpoons \mathrm{P}$, in which $\preceq$ is a universal homogeneous partial order on $A$, and where $G$ is the group of $\preceq$-automorphisms of $A$.

By forcing, we will extend $\mathcal{N}$ by adding a new partition $T$ of $A$, and show that in the extension $\mathcal{N}[T], T$ is a P-finite collection of P-finite subsets of $A$. Clearly, the partial order $\preceq$ will witness that $A$ itself is not P-finite in $\mathcal{N}[T]$ (as well as in $\mathcal{N})$, so we will have that in $\mathcal{N}[T], A$ is a P-finite union of P-finite sets that is not P-finite.

Let $\mathbf{P}$ be the notion of forcing whose conditions are partitions of finite subsets of $A$ with the qualification that no two elements in the same part of any of these partitions are $\preceq$-comparable. We say that a condition $p$ extends a condition $q$ if and only if $\bigcup q \subseteq \bigcup p$ and the restriction of the partition $p$ to the set $\bigcup q$ is the partition $q$.

Let $\Gamma$ be a $\mathbf{P}$-generic filter over $\mathcal{N}$. There is a partition $T$ of $A$ defined by $\Gamma$ (and from which $\Gamma$ may be defined); say $a$ and $b$ are in the same part of $T$ iff there is a condition $p \in \mathbf{P}$ with $\{a, b\} \in p \in \Gamma$. For each $t \in T$, the elements of $t$ are pairwise $\preceq$-incomparable. For each $a \in A$, let $T_{a}$ denote the part such that $a \in T_{a} \in T$. Let $\dot{T}$ be a standard $\mathbf{P}$-name for $T$, and for each $a \in A$ let $\dot{T}_{a}$ be a standard $\mathbf{P}$-name for $T_{a}$. The theorem is concluded by proving the following two claims.

Claim A: For each $a \in A$, the set $T_{a}$ is P-finite in $\mathcal{N}[T]$.

Claim B: $T$ is P-finite in $\mathcal{N}[G]$.

(Proof of Claim A.) Fix $a \in A$, and let $\leq$ be a partial order on $T_{a}$. Suppose $\dot{\leq}$ is a $\mathbf{P}$-name for $\leq$, and let $E$ be a finite support for $\dot{\dot{x}}$ in $\mathcal{N}$. Let $n$ be the number of $E$-types (with respect to the universal homogeneous order $\preceq$ on $A$ ). We will show that there is a $\leq$-maximal element in $T_{a}$ by showing that any chain of elements of $T_{a}$ linearly ordered by $\leq$ has cardinality at most $n$.

So by way of contradiction, suppose there is a chain of elements in $T_{a}$ with more than $n$ elements. Since there are only $n E$-types, there must be distinct elements $b$ and $c$ in this chain with the same $E$-type, say with $b \leq c$. Let $p \in \mathbf{P}$ such that $p \Vdash b \dot{\leq} c$. Assume also that $p \Vdash\{b, c\} \subset \dot{T}_{a}$, which is to say that $\{a, b, c\}$ is a subset of some element of $p$. Since $b$ and $c$ are $\preceq$-incomparable (because they are both in $T_{a}$ ) and have the same $E$-type, there is an automorphism $\pi \in G$ such that $\pi$ fixes $E$ pointwise and $\pi$ switches $b$ and $c$. Furthermore, we can arrange so that $\bigcup(\pi p) \cap \bigcup p \subseteq E \cup\{b, c\}$ (Lemma $4(2)$ with $D=\bigcup p \backslash(E \cup\{b, c\})$ ). From this last remark and the fact that $b$ and $c$ are in the same part of $p$, it follows that $p$ and $\pi p$ are compatible. And yet $\pi p \Vdash \pi b \dot{\leq} \pi c$, which is to say $\pi p \Vdash c \dot{\leq} b$. This contradiction finishes the proof of the first claim.

(Proof of Claim B.) Let $\leq$ be a partial order on $T$ in $\mathcal{N}[T]$, and let $\dot{\leq}$ be a $\mathbf{P}$-name for $\leq$ supported by $E$ in $\mathcal{N}$. Let $T_{1}$ and $T_{2}$ be distinct elements of $T$, neither of which meet $E$; we will show that $T_{1}$ and $T_{2}$ cannot be comparable under 
$\leq$. By $\mathbf{P}$-genericity of $T$, there is some $b \in T_{1}$ and some $c \in T_{2}$ such that $b$ and $c$ are $\preceq$-incomparable and each of the same $E$-type.

Now suppose by way of contradiction that there is a condition $p \in \Gamma$ such that $p \Vdash \dot{T}_{b} \dot{\leq} \dot{T}_{c} \wedge \dot{T}_{b} \neq \dot{T}_{c}$. Since neither $T_{a}$ nor $T_{b}$ meet $E$ in $\mathcal{N}[T]$, we have that neither $b$ nor $c$ is in the same part of $p$ as any element of $E$. Again, we can find $\pi \in G$ such that $\pi$ fixes $E$ pointwise, $\pi$ switches $b$ and $c$, and such that $\bigcup(\pi p) \cap \bigcup p \subseteq E \cup\{b, c\}$. Again, $\pi p$ and $p$ must be compatible, yet $\pi p \Vdash \dot{T}_{c} \leq \dot{T}_{b}$, and we have a contradiction. Thus elements of $T$ that do not meet $E$ are $\leq$-incomparable. Since only finitely many elements of $T$ do meet $E$, there must be a $\leq$-maximal element in $T$.

\section{REFERENCES}

[bs89] A. Blass and A. Scedrov. Freyd's models for the independence of the axiom of choice. Memoirs of the American Mathematical Society, 79(104), 1989.

[rd72] R. Dedekind. Stetigkeit und irrationale Zahlen. Vieweg, Braunschweig, 1872.

[odlc02] O. De la Cruz. Finiteness and choice. Fundamenta Mathematicae, 173:57-57, 2002.

[hr98] P. E. Howard and J. E. Rubin. Consequences of the Axiom of Choice. American Mathematical Society, Providence, RI, 1998.

[hy89] P. E. Howard and M. F. Yorke. Definitions of finite. Fund. Math., 133(3):169-177, 1989.

[tjj73] T. J. Jech. The Axiom of Choice. North-Holland Publishing Co., Amsterdam, 1973. Studies in Logic and the Foundations of Mathematics, Vol. 75.

[js66] T. J. Jech and A. Sochor. Applications of the $\Theta$-model. Bulletin de l'Académie Polonaise des Sciences, 14:533-537, 1966.

[al58] A. Lévy. The independence of various definitions of finiteness. Fund. Math., 46:1-13, 1958.

[am38] A. Mostowski. Über den Begriff einer endlichen Menge. C. R. des Séances de la Société des Sciences et des Lettres de Varsovie, 31(Cl. III):13-20, 1938.

[at24] A. Tarski. Sur les ensembles finis. Fund. Math., 6:45-95, 1924.

[jt74] J. Truss. Classes of Dedekind finite cardinals. Fund. Math., 84(3):187-208, 1974.

(O. De la Cruz) Department of Mathematics, Purdue University, West Lafayette, IN 47907-1395, U.S.A., And Centre de Recerca Matemàtica, Barcelona, Spain.

E-mail address: odlc@math.purdue.edu

(D. Dzhafarov) Department of Mathematics, Purdue University, West Lafayette, IN 47907-1395, U.S.A.

E-mail address: ddzhafar@math.purdue.edu

(E. Hall) Department of Mathematics and Statistics, University of Missouri-Kansas City, Kansas City, MO, 64110-2499, U.S.A.

E-mail address: halle@umkc.edu 